\title{
Multi-layer observer as new structure for state estimation in linear systems
}

\author{
Jakub Bernat, Jakub Kolota, Paulina Superczyńska, SŁawomir StęPień \\ Faculty of Computing, Poznan University of Technology \\ Piotrowo 3a, 61-138 Poznań, Poland \\ email: jakub.bernat@put.poznan.pl
}

(Received: 29.09.2016, revised: 10.02.2017)

\begin{abstract}
A new structure to design observers for linear systems is presented in this work. The key step is the construction of two layers where the first consists of multiple observers and the second connects them providing the weighted estimation state. The main difficulty is to find a new feedback which is responsible for control weights. To define observation law, we rely on multi observers from the first layer. The proposed structure significantly improves the transient characteristics of the observation process, which is shown in illustrative examples.
\end{abstract}

Key words: Luenberger observer, Multi Observer, electric DC motor

\section{Introduction}

The observer design is an important problem in the control systems theory. The most popular observer has been proposed long time ago by Luenberger [7]. Currently the observation problem is solved for linear and nonlinear systems $[4,6,11]$. In this paper our goal is to improve transients in the observation process in the case of a linear system. Thus, we consider Luenberger based observers.

The crucial problem connected with the observer design is to achieve the desired performance. An attractive idea is to alter the observer gain, which has been analyzed in other works $[9,11-13]$.

Another approach is found in the work [2] where a plant is described by switching a finite set of models. This requires a switched observer structure. The set of observers is applied to provide the convergence of an estimation process despite time varying parameters. However in every time instant only one observer is chosen to estimate the state hence we can say nothing about a multi observer structure.

An interesting idea is to refine the structure of the observer. For example, in the work [10] the transient improvement is obtained by introducing the additional dynamics into the ob- 
server process. In this approach the static Luenberger observer is transformed into dynamic form by replacing a static gain within a dynamic gain.

The purpose of this paper is to define a new framework which may be called Multi Observer. The authors are partially motivated by an approach formulated for the adaptive control. In works [8] the indirect adaptive controller is designed by using the identification process relying on many models, which are working simultaneously.

In this paper, our goal is to refine a structure of the Luenberger observer by its extension from a single to a multiple observer. This produces two layers. The former is a set of Luenberger observers with different estimation states. The latter calculates the weights of the first layer observers. Thanks to the auxiliary layer, we are enabled to provide a new estimation law. The proposed technique may be applied to any kind of Luenberger observer, for instance reduced order observer. Furthermore, the authors already applied the Multi Observer structure to reduce a peaking phenomenon in High Gain Observers in work [3]. In the presented work, thanks to linearity of the Luenberger observer, we may solve the problem Multi Observer problem in a very elegant way. The improvement of observer process performance is examined by simulations. To show advantage of its application in the real world, we apply a Multi Observer to a sensorless problem of a DC motor. Relying on simulations we show that a Multi Observer is not significantly affected by noise.

\section{Multi observer definition}

We analyze the observer design technique for a multi input multi output linear system given by:

$$
\begin{gathered}
\frac{\mathrm{d} x}{\mathrm{~d} t}(t)=A x(t)+B u(t), \\
y(t)=C x(t),
\end{gathered}
$$

where: $x \in \boldsymbol{R}^{n}$ is the system state, $u \in \boldsymbol{R}^{m}$ is the input variable, $y \in \boldsymbol{R}^{p}$ is the output variable. The matrix $A \in \boldsymbol{R}^{n \times n}, B \in \boldsymbol{R}^{n \times m}, C \in \boldsymbol{R}^{p \times n}$ consists of known parameters. We assume that the system is observable.

A typical approach is to design a Luenberger observer, which consists of a single system:

$$
\begin{gathered}
\frac{\mathrm{d} \hat{x}}{\mathrm{~d} t}(t)=A \hat{x}(t)+B u(t)-L(\hat{y}(t)-y(t)), \\
\hat{y}(t)=C \hat{x}(t),
\end{gathered}
$$

where $L \in \boldsymbol{R}^{p \times n}$ is the gain matrix. Next, we obtain an autonomous system

$$
\frac{\mathrm{d} e}{\mathrm{~d} t}(t)=(A-L C) e(t),
$$

where $e(t)=\hat{x}(t)-x(t)$ is the estimation error. We assume that $A-L C$ has poles in the left plane. This is easy to obtain for any observable system by designing gain $L$. 
The above structure, where for a one plant is only one observer is common for the previous work. In our approach we would like to extend the number of observers to more than 1 . Hence we define $M$ observers

$$
\begin{gathered}
\frac{\mathrm{d} \hat{x}_{k}}{\mathrm{~d} t}(t)=A \hat{x}_{k}(t)+B u(t)-L\left(\hat{y}_{k}(t)-y(t)\right), \\
\hat{y}_{k}(t)=C \hat{x}_{k}(t),
\end{gathered}
$$

where $k=1 \ldots n+1$ is the observer index. It is worth noting that the difference between the observers is only between the initial state. Due to the introduction of many observers, we have many state vectors $\hat{x}_{k}$.

Nonetheless in the control system we require only one value of a state estimate. Thus, we combine all vectors from $M$ observers into one

$$
\hat{x}(t)=\sum_{k=1}^{n+1} \alpha_{k}(t) \hat{x}_{k}(t),
$$

where $\alpha_{k} \in \mathbf{R}$ represents weight coefficients. Please note that the proposed structure gives us a chance to estimate the observer state in two layers. The first is well known and is based on a Luenberger observer. The second is related to adjusting the influence of $k$ observer on the final result. Now, we may define our goal of this work. We would like to find how to define the observation law in the second layer.

\section{Main results}

In this section we would like to present two main statements. The first proves that there exists such $\alpha_{k}^{*}$ that estimates state $x$ through Equation (8). The second defines the mapping which enables us to construct an observer.

To build the observer we calculate the observation error for all models. The error is defined as:

$$
e_{\sigma}(t)=\hat{x}(t)-x(t)=\sum_{k=1}^{n+1} \alpha_{k}(t) \hat{x}_{k}(t)-x(t) .
$$

The error is found between the Multi Observer state $\hat{x}$ and the system state $x$. We assume that

$$
\sum_{k=1}^{n+1} \alpha_{k}(t)=1
$$

and hence 


$$
e_{\sigma}(t)=\sum_{k=1}^{n+1} \alpha_{k}(t) e_{k}(t)
$$

where $e_{k}$ is the single observer error, whose derivative is the same as in Equation (5)

$$
\frac{\mathrm{d} e_{k}}{\mathrm{~d} t}(t)=(A-L C) e_{k}(t) .
$$

Lemma 1. Suppose $\hat{x}_{k}(0)-x_{n+1}(0), k=1 \ldots n$ as linearly independent vectors. Then, there exist weights $\alpha_{k}^{*}, k=1 \ldots n+1$ which are satisfied by the equation

$$
x(0)=\sum_{k=1}^{n+1} \alpha_{k}^{*} \hat{x}_{k}(0) .
$$

Furthermore, if the equation

$$
x(0)=\sum_{k=1}^{n+1} \alpha_{k}(t) \hat{x}_{k}(0)
$$

is true, then $\alpha_{k}(t)=\alpha_{k}^{*}$.

Proof. With the help of (10), we consider the following equation

$$
x(0)=\sum_{k=1}^{n+1} \alpha_{k}(t) \hat{x}_{k}(0)=\hat{x}_{n+1}(0)+\sum_{k=1}^{n} \alpha_{k}(t)\left[\hat{x}_{k}(0)-\hat{x}_{M}(0)\right] .
$$

Because $\hat{x}_{k}(0)-x_{n+1}(0), k=1 \ldots n$ are linearly independent vectors, we can choose $\alpha_{k}^{*}$ to span any vector in $\boldsymbol{R}^{n \times 1}$. Hence, we can choose such $\alpha_{k}^{*}$ that (15) is equal to $x(0)$. This assures that (13) is true.

To show second statement, we consider the following equation

$$
x(0)=\hat{x}_{n+1}(0)+\sum_{k=1}^{n} \alpha_{k}(t)\left[\hat{x}_{k}(0)-\hat{x}_{M}(0)\right] .
$$

Because of linearly independent vectors $\hat{x}_{k}(0)-\hat{x}_{n+1}(0)$, we conclude that $\alpha_{k}(t)=\alpha_{k}^{*}$.

Lemma 2. Under the foregoing conditions, the observation error $e_{\sigma}$ is 0 for $t \rightarrow \infty$ if and only if $\alpha_{k}(t)=\alpha_{k}^{*}$.

Proof. Let consider $\alpha_{k}(t)=\alpha_{k}^{*}$. Then

$$
e_{\sigma}(t)=\hat{x}(t)-x(t)=\sum_{k=1}^{n+1} \alpha_{k}^{*} \hat{x}_{k}(t)-x(t)
$$

The solution of equation error (12) for $k$ observer is given by 


$$
e_{k}(t)=\Phi(t) e_{k}(0)
$$

where $\Phi(t)$ is the state-transition matrix. Hence, with the help of Lemma 1 we obtain:

$$
e_{\sigma}(t)=\Phi(t)\left[\sum_{k=1}^{n+1} \alpha_{k}^{*} \hat{x}_{k}(0)-x(0)\right]=0,
$$

which finishes the first part of the proof.

If we consider $e_{\sigma}(t)=0$, then

$$
\Phi(t)\left[\sum_{k=1}^{n+1} \alpha_{k}(t) \hat{x}_{k}(0)-x(0)\right]=0 .
$$

Because $\Phi(t)$ is invertible for $t>0$ then

$$
\sum_{k=1}^{n+1} \alpha_{k}(t) \hat{x}_{k}(0)-x(0)=0 .
$$

From Lemma 1, we conclude that $\alpha_{k}(t)=\alpha_{k}^{*}$.

From the point of view of the observation process, we search an algorithm which could estimate $\alpha_{k}(t)$ such that they produce $e_{\sigma}(t)=0$. It could be expected that if it is possible to estimate $\alpha_{k}(t)$ coefficients simultaneously with decaying $e_{k}(t)$, then the observation process will converge faster. Unfortunately, in Equation (11) error $e_{k}(t)$ is unknown in the observation process, hence we require additional mapping to obtain accessible signals. Let us consider the most obvious case

$$
\eta_{k}(t)=C e_{k}(t)
$$

and the following mapping:

$$
\eta(t)=\alpha_{1}(t) \eta_{1}(t)+\ldots+\alpha_{k}(t) \eta_{k}(t)+\ldots+\alpha_{n+1}(t) \eta_{n+1}(t) .
$$

Transform $\eta(t)=C e_{\sigma}(t)$ is not invertible. Therefore $\eta(t)=0$ does not ensure that $e_{\sigma}(t)$ is equal to 0 . This motivated us to search more sophisticated transformation. We calculate $n-1$ derivatives of (7)

$$
\begin{gathered}
\frac{\mathrm{d} \eta_{k}}{\mathrm{~d} t}(t)=C \frac{\mathrm{d} e_{k}}{\mathrm{~d} t}(t)=C A e_{k}(t)-C L \eta_{k}(t), \\
\frac{\mathrm{d}^{2} \eta_{k}}{\mathrm{~d} t^{2}}(t)=C A^{2} e_{k}(t)-C A L \eta_{k}(t)-C L \frac{\mathrm{d} \eta_{k}}{\mathrm{~d} t}(t), \\
\quad \vdots \\
\frac{\mathrm{d}^{n-1} \eta_{k}}{\mathrm{~d} t^{n-1}}(t)=C A^{n-1} e_{k}(t)-\ldots-C L \frac{\mathrm{d}^{n-2} \eta_{k}}{\mathrm{~d} t^{n-2}}(t) .
\end{gathered}
$$


The above equations can be integrated $n-1$ times and they can be expressed as a matrix equation

$$
\xi_{k}(t)=\left[\begin{array}{c}
C \\
C A \\
\vdots \\
C A^{n-1}
\end{array}\right] \underbrace{\int_{0}^{t} \ldots \int_{0}^{t} e(\tau) d \tau}_{n-1},
$$

where $\xi_{k} \in \boldsymbol{R}^{n \times 1}$ is defined as

$$
\xi_{k}(t)=\left[\begin{array}{cccc}
1 & 0 & \ldots & 0 \\
C L & 1 & \ldots & 0 \\
\vdots & \vdots & \ddots & \vdots \\
C A^{n-2} L & C A^{n-1} L & \ldots & 1
\end{array}\right]\left[\begin{array}{c}
v_{n-1, k}(t) \\
\vdots \\
v_{0, k}(t)
\end{array}\right]
$$

The signals $v_{j, k}(t) \in \mathbf{R}$, where $j$ denotes an index and $k$ is the observer number, create the integration chain

$$
\begin{gathered}
v_{0, k}(t)=\eta_{k}(t), \\
\frac{\mathrm{d} v_{1, k}(t)}{\mathrm{d} t}=v_{0, k}(t), \\
\frac{\mathrm{d} v_{2, k}(t)}{\mathrm{d} t}=v_{1, k}(t), \\
\vdots \\
\frac{\mathrm{d} v_{n-1, k}(t)}{\mathrm{d} t}=v_{n-2, k}(t) .
\end{gathered}
$$

It is worth to note that $\xi_{k}(t)$ is found relying on output error $\eta_{k}(t)$ and its integrals. Hence, it is easily accessible in the control system. Now, we are ready to state a theorem which enables estimation of $\alpha_{k}(t)$ coefficients.

Theorem 1. Let consider $\xi_{k}(t)$ defined by (27) and (28-32) and introduce an auxiliary error

$$
e_{\xi}(t)=\sum_{k=1}^{n+1} \alpha_{k}(t) \xi_{k}(t)
$$

then, for $t>0$ auxiliary error $e_{\xi}(t)$ is equal to 0 if and only if $\alpha_{k}(t)=\alpha_{k}^{*}$.

Proof. Let consider $\alpha_{k}(t)=\alpha_{k}^{*}$. Thanks to $e_{k}(t)=\Phi(t) e_{k}(0)$ the solution on $\xi_{k}(t)$ is equal to 


$$
\xi_{k}(t)=\left[\begin{array}{c}
C \\
C A \\
\vdots \\
C A^{n-1}
\end{array}\right] \Gamma(t) e_{k}(0)
$$

where $\Gamma(t)=\underbrace{\int_{0}^{t} \ldots \int_{0}^{t} \Phi(\tau) d \tau}_{n-1}$. The auxiliary error may be rewritten as

$$
e_{\xi}(t)=\left[\begin{array}{c}
C \\
C A \\
\vdots \\
C A^{n-1}
\end{array}\right] \Gamma(t) \sum_{k=1}^{M} \alpha_{k}(t) e_{k}(0)
$$

and with the help of (10)

$$
e_{\xi}(t)=\left[\begin{array}{c}
C \\
C A \\
\vdots \\
C A^{n-1}
\end{array}\right] \Gamma(t)\left[\sum_{k=1}^{n+1} \alpha_{k}(t) \hat{x}_{k}(0)-x(0)\right] .
$$

From Lemma (1), we conclude that $e_{\xi}(t)=0$. This finishes the first part of the proof. Now, let assume that $e_{\xi}(t)=0$. This yields to

$$
\left[\begin{array}{c}
C \\
C A \\
\vdots \\
C A^{n-1}
\end{array}\right] \Gamma(t)\left[\sum_{k=1}^{n+1} \alpha_{k}(t) \hat{x}_{k}(0)-x(0)\right]=0 \text {. }
$$

From the properties of the transition matrix we known that $\Gamma(t)$ is invertible for $t>0$. Additionally, we have also assumed that the matrix is observable, hence $\left[\begin{array}{llll}C & C A & \ldots & C A^{n-1}\end{array}\right]$ is invertible. Thus, we conclude that

$$
\sum_{k=1}^{n+1} \alpha_{k}(t) \hat{x}_{k}(0)-x(0)=0
$$

and from Lemma $\alpha_{k}(t)=\alpha_{k}^{*}$.

The above result describes how to estimate state $x(t)$ in the second layer. The key-note of the estimation algorithm is to find $\alpha_{k}^{*}$ whose value is unknown at the beginning of the observation process. As soon as we estimate $\alpha_{k}^{*}$, we known from Theorem 1 that $e_{\sigma}(t)$ is reduced 
to 0 . This means that $\hat{x}(t)$ is equal to $x(t)$. In the next section we will show how to find $\alpha_{k}^{*}$ based on (33).

Now, the main point of applying a Multi Observer is clearly visible. It has to be emphasized that the goal of using many observers is not to find the initial starting guest of $\hat{x}(0)$ as close as $x(0)$. The second layer is introduced to provide additional mapping and thereby expand the capabilities of the observation process.

\section{Estimation algorithm}

In this section we describe a method of estimation of weights $\alpha_{k}(t)$. Relying on Theorem 2 we would like to find $\alpha_{k}^{*}$ which reduces $e_{\xi}(t)=0$. Hence, with the help of (10) it is worth to rewrite (33) as

$$
e_{\xi}(t)=\alpha_{n+1} \xi_{n+1}(t)+\sum_{k=1}^{n} \alpha_{k} \xi_{k}(t)=\xi_{n+1}(t)+\sum_{k=1}^{n} \alpha_{k}\left[\xi_{k}(t)-\xi_{n+1}(t)\right] .
$$

The simplest method to find $\alpha_{k}^{*}$ is to solve the above equation such that $e_{\xi}(t)=0$. However, such a solver is difficult to implement in a real time system and the disturbances will greatly influence the solution. Hence, we propose to find $\alpha_{k}(t)$ coefficients by an RLS algorithm which works in a discrete domain.

Let consider that the second layer works in the sampled time frame. We assume that the weight factors $\hat{\alpha}_{k}\left(n_{p} T_{p}\right)$ and integration chain $\xi_{k}\left(n_{p} T_{p}\right)$ are sampled and available as discrete signals. This means that the observers from the first layer and the integration chain (27) work in a continuous domain. In the considered problem, the regressor is specified as

$$
y_{r l s}\left[n_{p}\right]=\varphi_{r l s}^{T}\left[n_{p}\right] \hat{\theta}_{r l s}\left[n_{p}\right]+\varepsilon_{r l s}\left[n_{p}\right],
$$

where: $\hat{\theta}_{r l s} \in \mathbf{R}^{n \times 1}$ is the vector with estimated parameters, $\varphi_{r l s} \in \boldsymbol{R}^{n \times n}$ is the vector of known functions and $y_{r l s} \in \boldsymbol{R}^{n \times 1}$ is the output. The above vectors are defined as

$$
\begin{aligned}
& \theta_{r l s}\left[n_{p}\right]=\left[\begin{array}{llll}
\alpha_{1}\left(n_{p} T_{p}\right) & \alpha_{2}\left(n_{p} T_{p}\right) & \ldots & \alpha_{n}\left(n_{p} T_{p}\right)
\end{array}\right]^{T}, \\
& \varphi_{r l s}\left[n_{p}\right]=\left[\begin{array}{c}
\xi_{1}\left(n_{p} T_{p}\right)-\xi_{M}\left(n_{p} T_{p}\right) \\
\vdots \\
\xi_{M-1}\left(n_{p} T_{p}\right)-\xi_{M}\left(n_{p} T_{p}\right)
\end{array}\right]^{T}, \\
& y_{r l s}\left[n_{p}\right]=\left[-\xi_{M}\left(n_{p} T_{p}\right)\right] \text {. }
\end{aligned}
$$

Please note that if an estimator reduces $\varepsilon_{r l s}$ to 0 then from Equation (33) we conclude that $e_{\xi}$ is also equal to 0 . This means that $\alpha_{k}(t)=\alpha_{k}^{*}$ and hence the estimated state $x(t)$ is equal to $x(t)$. 
The control theory provides many algorithms for solving an estimation problem based on regressor. In this work we have chosen an RLS algorithm. Relying on [1], we apply the following estimator

$$
\begin{gathered}
K\left[n_{p}\right]=P\left[n_{p}-1\right] \varphi_{r l s}\left[n_{p}\right]\left(I+\varphi_{r l s}^{T}\left[n_{p}\right] P\left[n_{p}-1\right] \varphi_{r l s}\left[n_{p}\right]\right)^{-1}, \\
P\left[n_{p}\right]=\left(I-K\left[n_{p}\right] \varphi_{r l s}^{T}\left[n_{p}\right]\right)^{-1} P\left[n_{p}-1\right], \\
\hat{\theta}_{r l s}\left[n_{p}\right]=\hat{\theta}_{r l s}\left[n_{p}-1\right]+K\left[n_{p}\right]\left(y_{r l s}\left[n_{p}\right]-\varphi_{r l s}^{T}\left[n_{p}\right] \hat{\theta}_{r l s}\left[n_{p}-1\right]\right),
\end{gathered}
$$

where: $I \in \boldsymbol{R}^{n \times n}$ is the identity matrix, $K \in \boldsymbol{R}^{n \times n}$ is the auxiliary gain and $P \in \boldsymbol{R}^{n \times n}$ and $P=p_{r l s} I$ with $p_{r l s}>0$. The specified estimator error converges to 0 because matrix $\varphi_{r l s}$ is full rank.

In the next section, we will show that the proposed methodology provides great transient improvement in the observation process.

\section{Simulation}

\subsection{Compare with Luenberger Observer}

To show the properties of Multi Observer, we consider a linear system defined as

$$
\begin{gathered}
A=\left[\begin{array}{ccc}
0 & 1 & 0 \\
0 & 0 & 1 \\
-a_{2} & -a_{1} & -a_{0}
\end{array}\right], B=\left[\begin{array}{l}
0 \\
0 \\
1
\end{array}\right], \\
C=\left[\begin{array}{lll}
1 & 0 & 0
\end{array}\right],
\end{gathered}
$$

where $a_{0}=-263.5, a_{1}=-129.1, a_{2}=-20.1$. The single observer gains are set to

$$
\begin{aligned}
L_{1} & =\left[\begin{array}{lll}
-8.1 & 81.6 & -794.5
\end{array}\right], \\
L_{2} & =\left[\begin{array}{lll}
39.9 & 243.8 & -2816
\end{array}\right], \\
L_{3} & =\left[\begin{array}{lll}
99.9 & 2637.5 & -3188.8
\end{array}\right],
\end{aligned}
$$

and hence $A-L_{j} C, j=1 \ldots 3$ poles are equal to

$$
\begin{aligned}
& p_{1}=\left[\begin{array}{lll}
-3.5 & -4 & -4.5
\end{array}\right], \\
& p_{2}=\left[\begin{array}{lll}
-25 & -20 & -15
\end{array}\right], \\
& p_{3}=\left[\begin{array}{lll}
-45 & -40 & -35
\end{array}\right] .
\end{aligned}
$$


We consider three different gains in a single observer to show the influence of Multi Observer on an observation process. Now, we define the number of a multiple observer equal to $n+1=4$. The initial estimation points are equal to:

$$
\hat{x}_{1}=(0)=\left[\begin{array}{l}
4 \\
0 \\
0
\end{array}\right], \quad \hat{x}_{2}=(0)=\left[\begin{array}{l}
0 \\
4 \\
0
\end{array}\right], \quad \hat{x}_{3}=(0)=\left[\begin{array}{l}
0 \\
0 \\
4
\end{array}\right], \quad \hat{x}_{4}=(0)=\left[\begin{array}{l}
0 \\
0 \\
0
\end{array}\right]
$$

and weight's coefficients are given by:

$$
\alpha_{1}(0)=\ldots=\alpha_{4}(0)=0.25 .
$$

Thus, the initial estimation point is:

$$
x(0)=\left[\begin{array}{lll}
1 & 1 & 1
\end{array}\right]^{T} .
$$

The RLS gain is set to $p_{r l s}=10$. The plant and observer have been simulated by the Eulerforward method with sampling time $T_{p}=1 \mathrm{~ms}$. The output signal has been disturbed by white noise with variance 0.05 . The input signal is square waveform with an amplitude of 4 and a period of $250 \mathrm{~ms}$. The results are presented in Fig. 1. It is visible that for all gains $L_{1}, L_{2}$ and $L_{3}$ Multi Observer has a smaller overshoot and faster converge time than the single observer. It also does not increase the level of noise in estimated states. It is visible that for high gain values, a single observer has peaks at the beginning of transients. This effect does not take place for a Multi Observer.

\subsection{Observer for speed-sensorless DC}

Let consider an observer applied to a constructed speed-sensorless DC drive [4]. We assume a DC motor model as

$$
\begin{aligned}
\frac{d \omega}{d t} & =-\frac{b}{J} \omega+\frac{k_{t}}{J} i-\frac{1}{J} T_{L}, \\
\frac{d i}{d t} & =-\frac{R}{L} i-\frac{k_{t}}{L} \omega+\frac{1}{L} u,
\end{aligned}
$$

where: $\omega$ is the motor speed, $i$ is the phase current, $T_{L}$ is the constant load torque, $u$ is the phase voltage, $R$ is the resistance, $L$ is the inductance, $k_{t}$ is the torque constant, $b$ is the viscous friction and $J$ is the rotor inertia. Motor parameters are defined in Table 1.

Table 1. Motor DC parameters

\begin{tabular}{l|c|c|c}
\hline Parameter name & Symbol & Value & Unit \\
\hline Resistance & $R$ & 3.2 & $\Omega$ \\
\hline Inductance & $L$ & 8.6 & $\mathrm{mH}$ \\
\hline Torque constant & $k_{t}$ & 0.0319 & $\mathrm{Nm} / \mathrm{A}$ \\
\hline Viscous friction & $b$ & 0.00012 & $\mathrm{Nms}$ \\
\hline Rotor inertia & $J$ & $30 \cdot 10^{-6}$ & $\mathrm{~kg} \cdot \mathrm{m}^{2}$ \\
\hline
\end{tabular}




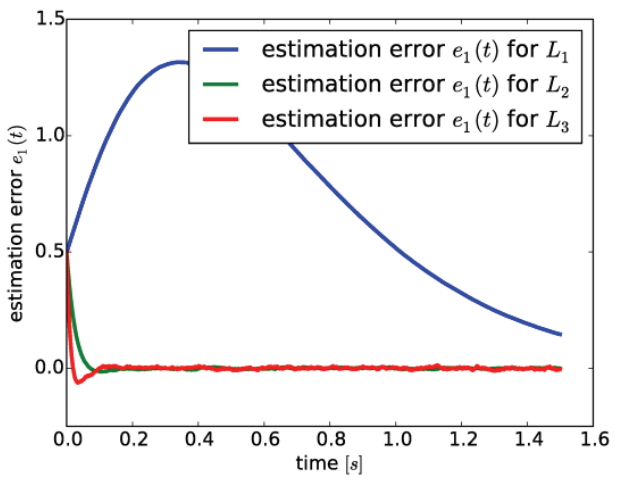

(a) single observer

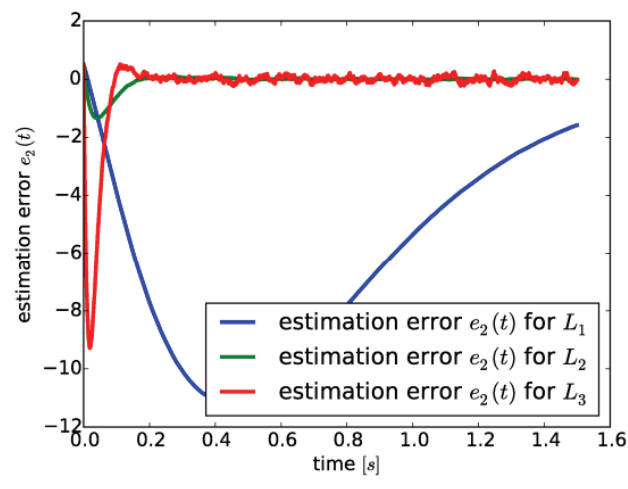

(c) single observer

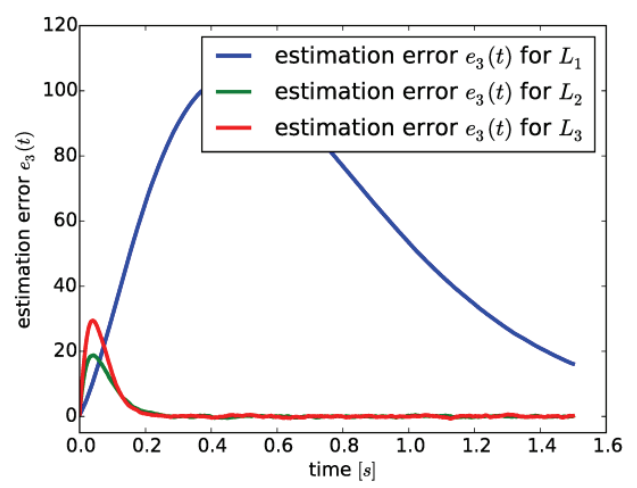

(e) single observer

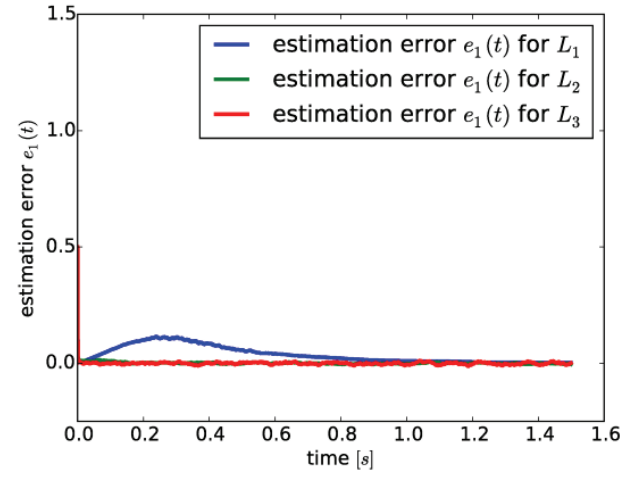

(b) multi observer

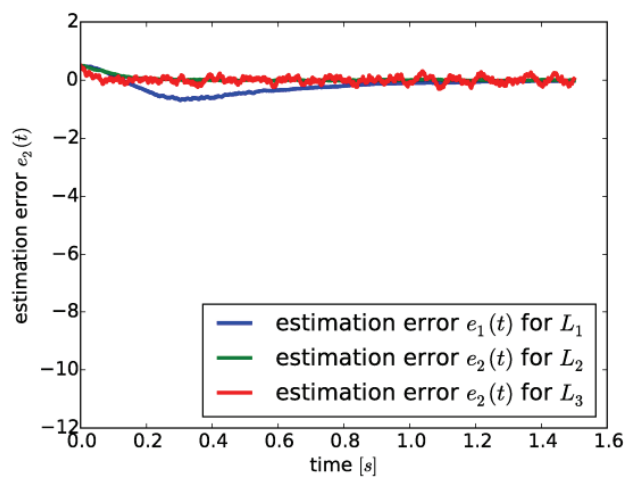

(d) multi observer

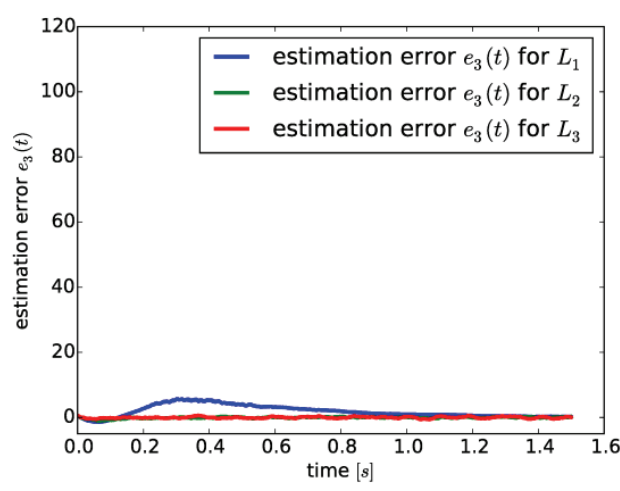

(f) multi observer

Fig. 1. Converge of error estimation for Multi Observer (a, c, e) and single observer (b, d, f)

In the speed-sensorless problem we accept that motor speed and load torque are inaccessible. Hence, it is useful to consider load torque as motor state variable. This gives possibility to estimate its value. Thus, we transform model (58-59) into state space system (1) 


$$
\begin{gathered}
x=\left[\begin{array}{ccc}
\omega & i & T_{L}
\end{array}\right]^{T}, y=[i], u=[u], \\
A=\left[\begin{array}{ccc}
-\frac{b}{J} & \frac{k_{t}}{J} & -\frac{1}{J} \\
-\frac{k_{t}}{L} & -\frac{R}{L} & 0 \\
0 & 0 & 0
\end{array}\right], B=\left[\begin{array}{c}
0 \\
\frac{1}{L} \\
0
\end{array}\right], \\
C=\left[\begin{array}{lll}
0 & 1 & 0
\end{array}\right] .
\end{gathered}
$$

Now, to construct an observer we choose matrix $L$ as

$$
L=\left[\begin{array}{lll}
0 & 0 & -\mu
\end{array}\right]
$$

which gives the following linear time-invariant system

$$
\frac{\mathrm{d}}{\mathrm{d} t}\left[\begin{array}{c}
e_{\omega} \\
e_{i} \\
e_{T}
\end{array}\right]=\left[\begin{array}{ccc}
-\frac{b}{J} & \frac{k_{t}}{J} & -\frac{1}{J} \\
-\frac{k_{t}}{L} & -\frac{R}{L} & 0 \\
0 & \mu & 0
\end{array}\right]\left[\begin{array}{c}
e_{\omega} \\
e_{i} \\
e_{T}
\end{array}\right]
$$

where: $e_{\omega}=\hat{\omega}-\omega, e_{i}=\hat{i}-i$ and $e_{T}=\hat{T}_{L}-T_{L}$ represent the estimated speed error, estimated current error and estimated load torque error respectively. We set $\mu$ equal to $0.06 \mathrm{Nm} / \mathrm{As}$. Thus, system (64) has poles equal to $-361.11,-13.46,-1.53$.

In our system, load torque is modelled as a state variable. This provides that the observer is represented by $A, C$ and $L$ matrixes of system (3). Now, we can apply a Multi Observer extension. The number of single observers is equal to $n+1=4$. Each observer has a different initial point

$$
\hat{x}_{1}(0)=\left[\begin{array}{c}
400 \\
0 \\
0
\end{array}\right] \quad \hat{x}_{2}(0)=\left[\begin{array}{l}
0 \\
4 \\
0
\end{array}\right] \quad \hat{x}_{3}(0)=\left[\begin{array}{c}
0 \\
0 \\
0.08
\end{array}\right] \quad \hat{x}_{4}(0)=\left[\begin{array}{l}
0 \\
0 \\
0
\end{array}\right]
$$

The above points satisfies condition (13). We choose initial weights coefficients equal to

$$
\alpha_{1}(0)=\ldots=\alpha_{4}(0)=0.25
$$

and hence the initial estimated state is given by $x(0)=\left[\begin{array}{lll}100 & 1 & 0.02\end{array}\right]^{\mathrm{T}}$. The RLS algorithm gain is set to $p_{r l s}=50$. To execute simulation, the motor model and Multi Observer are approximated by the Euler-forward method with sampling time $T_{p}=0.1 \mathrm{~ms}$. The motor voltage is equal to $10 \mathrm{~V}$ and load torque is equal to $0.01 \mathrm{Nm}$. To imitate real environment, white noise is added to the output of the motor model with a variance of 0.05 . The simulation results are presented in Figs. 2, 3 and 4. Fig. 5 shows the transients of weights $\alpha_{k}$ which are 
implemented only in the Multi Observer case.The figures consist of the motor state and estimated state by Multi Observer (MO) and the single observer (SO). The latter is a Luenberger observer defined by $A, C$ and $L$ matrixes. It is visible that the Multi Observer converges faster than the single observer in all the cases. It has also a small overshoot. The estimated speed and motor current are also not affected by noise.

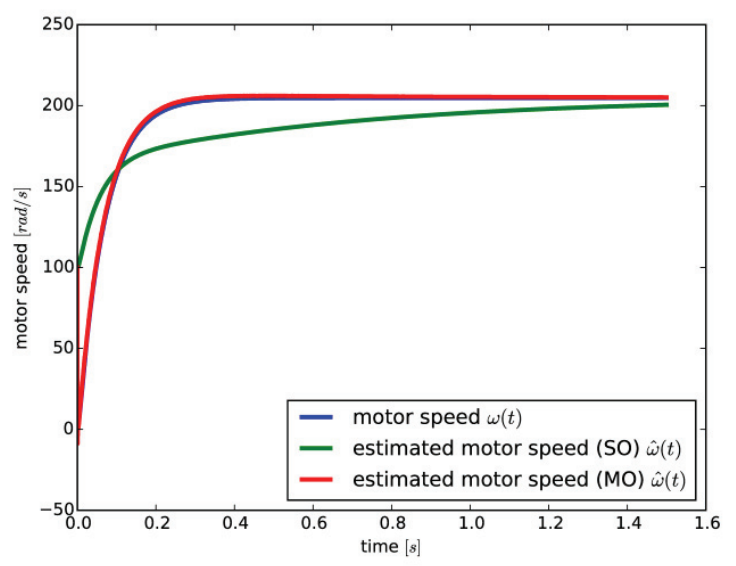

Fig. 2. Convergence of motor speed, estimated speed by single observer (SO) and Multi Observer (MO)

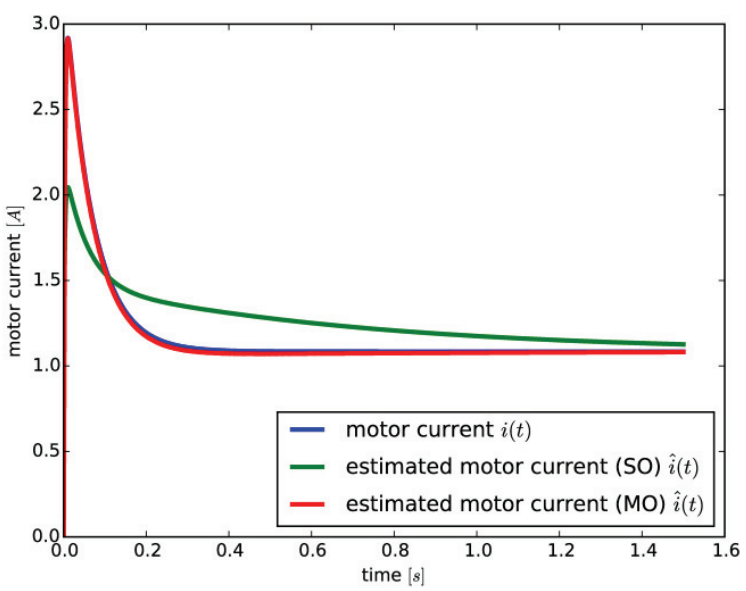

Fig. 3. Convergence of estimated motor current by single observer (SO) and Multi Observer (MO)

\section{Conclusions}

In this work a new framework has been proposed to solve the observation problem for a linear system. Based on the presented simulation, we have shown that it significantly improves transients. The analysis of properties of Multi Observer was performed as well. The future work is to apply the Multi Observer to real application. 


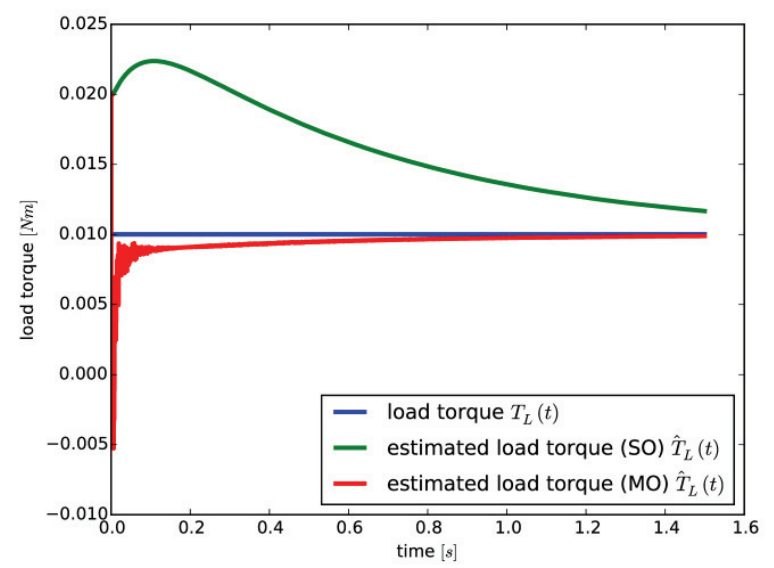

Fig. 4. Convergence of estimated load torque by single observer (SO) and Multi Observer (MO)

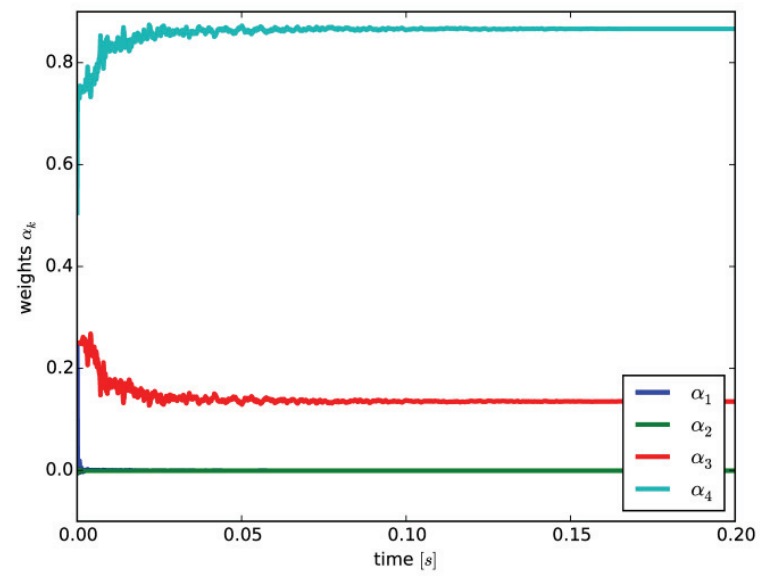

Fig. 5. Transients of Multi Observer weights

\section{References}

[1] Astrom K.J., Wittenmark B., Adaptive Control, Prentice Hall, New Jersey (1994).

[2] Bejarano F.J., Pisano A., Switched observers for switched linear systems with unknown inputs, IEEE Trans. on Automatic Control, vol. 56, no. 3, pp. 681-686 (2011).

[3] Bernat J., Stępień S., Multi-modelling as new estimation schema for high-gain observers, International Journal of Control, vol. 88, no. 6, pp. 1209-1222 (2015).

[4] Bernat J., Kolota J., Sensorless position estimator applied to nonlinear IPMC model, Smart Materials and Structures, vol. 25, no. 11 (2016).

[5] Bowes S.R., Sevinc A., Holliday D., New natural observer applied to speed-sensorless dc servo and induction motor, IEEE Transactions On Industrial Electronics, vol. 51, no. 5, pp. 1025-1032 (2004).

[6] Laidi K., Benmansour K., Ferdjouni A., Bouchhida O., Real-time implementation of an interconnected observer design for p-cells chopper, Archives of Electrical Engineering, vol. 59, no. 1-2, pp. 5-20 (2010) 
[7] Luenberger D.G., An introduction to observers, IEEE Trans. on Automatic Control, vol. 16, no. 6 , pp. 596-602 (1971).

[8] Narendra K.S., Han Z., New concepts in adaptive control using multiple models, IEEE Transactions on Automatic Control, vol. 57, no. 1, pp. 78-88 (2012).

[9] O’Reilly J., Observer for linear systems, Academic Press, London (1983).

[10] Park J.K., Shin D.R., Chung T.M., Dynamic observers for linear time-invariant systems, Automatica, vol. 38, pp. 1083-1087 (2002).

[11] Stocks M., Medvedev A., Linear-quadratic optimal time-varying observers with time-varying guaranteed convergence rate, IET Control Theory Appl., vol. 3, no. 2, pp. 181-188 (2009).

[12] Urbański K., Sensorless control of SRM at medium speed range, Archives of Electrical Engineering, vol. 60, no. 2, pp. 179-185 (2011).

[13] Wanga Z.B., Huang H.U., Robust h1 observer design of linear time-delay systems with parametric uncertainty, System and control letters, vol. 42, no. 1, pp. 303-312 (2001).

[14] Yao Y., Radun A.V., Proportional integral observer design for linear systems with time delay, IET Control Theory Appl. vol. 1, no. 4, pp. 887-892 (2007). 